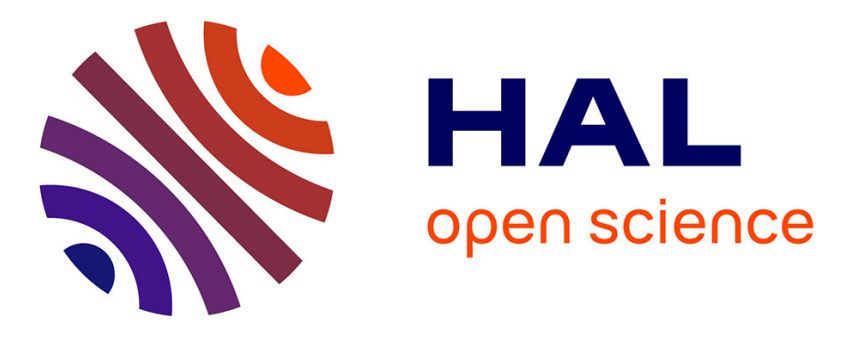

\title{
Actuator faut estimation based adaptive polytopic observer for a class of LPV descriptor systems
}

Mickael Rodrigues, Hamdi Habib, Didier Theilliol, Chokri Mechmeche, Naceur Benhadj Braiek

\section{- To cite this version:}

Mickael Rodrigues, Hamdi Habib, Didier Theilliol, Chokri Mechmeche, Naceur Benhadj Braiek. Actuator faut estimation based adaptive polytopic observer for a class of LPV descriptor systems. International Journal of Robust and Nonlinear Control, 2015, 25 (5), pp.673-688. 10.1002/rnc.3236 . hal-01058429

\section{HAL Id: hal-01058429 \\ https://hal.science/hal-01058429}

Submitted on 9 Sep 2014

HAL is a multi-disciplinary open access archive for the deposit and dissemination of scientific research documents, whether they are published or not. The documents may come from teaching and research institutions in France or abroad, or from public or private research centers.
L'archive ouverte pluridisciplinaire HAL, est destinée au dépôt et à la diffusion de documents scientifiques de niveau recherche, publiés ou non, émanant des établissements d'enseignement et de recherche français ou étrangers, des laboratoires publics ou privés. 


\title{
Actuator Faut Estimation based Adaptive Polytopic Observer for a Class of LPV Descriptor Systems
}

\author{
M. Rodrigues*, H. Hamdi**, D. Theilliol ${ }^{* * *}$, C. Mechmeche**and N. BenHadj Braiek** \\ *Université de Lyon, Lyon, F-69003, France ; Université Lyon 1, CNRS, UMR 5007, Laboratory of Automatic and \\ Process Control, LAGEP, Villeurbanne,F-69622,France.e-mail:mickael.rodrigues@univ-lyon1.fr \\ **Laboratory for Advanced Systems at the Tunisia Polytechnic School, University of Carthage, Tunisia. \\ *** Centre de Recherche en Automatique de Nancy (CRAN), Université de Lorraine, CNRS, B.P. 70239 F-54506 \\ Vandouvre lès Nancy, France.e-mail: didier.theilliol@univ-lorraine.fr
}

\begin{abstract}
SUMMARY
This paper presents an Adaptive Polytopic Observer (APO) design in order to develop an actuator fault estimation method dedicated to polytopic Linear Parameter Varying (LPV) descriptor systems. This paper extends a Fault Diagnosis (FD) method developed for regular LTI systems to polytopic LPV descriptor systems. Here, time-varying actuator faults are also considered whereas in many papers actuator faults are generally assumed to be constant. The design and convergence conditions of this APO are provided. The design is formulated through Linear Matrix Inequalities (LMI) techniques under equality constraints. The performances of the proposed actuator fault estimation scheme are illustrated using an electrical circuit.
\end{abstract}

Received ...

KEY WORDS: Adaptive Polytopic Observer, LPV descriptor systems, Actuator Fault Estimation, LMI

\section{INTRODUCTION}

During the last three decades, both theoretical and experimental researches on fault detection have been intensively developed. For obvious economical reasons, systems in the real world have to work perfectly at all time under all conditions. That's why it is crucial to be able to detect and identify the possible faults that may affect a system as early as possible in order to prevent significant performance degradations or damages of the system [6], [34]. Fault Detection and Diagnosis (FDD) has been well studied for physical plants which were modeled by T-S fuzzy models. Recently Such efforts studded by Jiang B. et al. [17], have led to the development of a method for integrated robust fault estimation and accommodation for a class of discrete-time nonlinear systems described by a T-S fuzzy model. Also, dynamic output feedback fault-tolerant controllers have been developed by Zhang K. et al. [31] for T-S fuzzy systems with actuator faults in which a fuzzy augmented fault observer is proposed to yield fault estimates and, based on the information of on-line fault estimates, observer based output feedback fault-tolerant controllers are designed. Nevertheless for nonlinear systems it still remain a challenge [22].

By the way, some techniques have been developed to approximate nonlinear systems by Linear Parameter Varying (LPV) models that can be used efficiently to represent nonlinear systems as in [4], [25] for a winding machine, [26] for a twin rotor system. These methods allow to apply powerful linear design tools to complex nonlinear models. Based on this LPV representation

*Correspondence to: M. Rodrigues Université de Lyon, Lyon, F-69003, France ; université Lyon 1, CNRS, UMR 5007, LAGEP, Villeurbanne, F-69622, France ; ESCPE, Villeurbanne, F-69622, France e-mail: mickael.rodrigues@ univlyon1.fr 
of nonlinear systems, some researchers from the FDI community have developed model-based methods using LPV ordinary models as in [2], [5], and [11]. Similar result is found in [29] for linear parameter-varying (LPV) systems the presented method is based on multi-objective $H_{-} / H_{\infty}$ fault detection observer design. LPV sliding mode observers have been largely introduced by [1] so as to reconstruct the states of the system. A particular class of LPV systems is the polytopic LPV form which allows to describe the system as a convex combination of sub-models defined by the vertices of a convex polytope [13], [15], [12]. These sub-models are then combined by convex weighting functions that yield to a global model.

Generally speaking, most of control and fault diagnosis methods for physical systems, use normal (or regular) models i.e. there is no algebraic relations between the system variables. However, Differential-Algebraic Equations (DAE) or implicit systems or singular systems or descriptor systems are of quite importance for the physical representation of some systems [20], [10]. Such systems appear for example in electrical circuits, mechanical systems with holonomic or non holonomic constraints, robotic systems with kinematical constraints and chemical systems [21]. Some practical problems must take into account physical constraints or algebraic relations and more generally impulsive behaviors caused by an improper transfer matrix: see the following books on singular systems [8], [9].

Various observer-based fault diagnosis methods for descriptor systems have been proposed but generally for Linear Time Invariant models [27]. The author in [19] studies an observer coming from a continuous nonlinear descriptor systems via a convex optimization. Recently, a fault diagnosis method to detect and estimate actuator faults for multi-models descriptor systems represented by using unknown input observers [15]. Therefore, in [3] the authors have presented a method of fault estimation for a particular class of discrete-time LPV descriptor systems. The authors in [16] and [14], have proposed a polytopic unknown inputs and proportional integral observers for LPV descriptor systems respectively. These observers are used to detect and isolate actuator faults. However, all these observers can only estimate constant faults which is a restrictive condition.

In order to estimate both constant and time varying faults, an Adaptive Polytopic Observer is proposed in this paper. In many applications where the parameters are unknown and states are not accessible, adaptive observer appears to be a valuable method in estimation of both parameters and states of the system. In [28] the authors have presented an adaptive fault diagnosis observer approach dedicated to regular LTI systems which can detect and estimate constant faults. In [32], the authors have designed an adaptive observer so as to estimate time-varying faults but only for regular LTI systems.

Thus, this paper proposes an Adaptive Polytopic Observer (APO) for a class of LPV descriptor systems. Fault Diagnosis for descriptor LPV system still remains a challenge and as far as authors knows there are very few papers for such systems that consider time-varying actuator fault estimation under disturbances. In literature, most of fault diagnosis approaches are designed only for regular systems and not for descriptor systems. To tackle such problem, the entire development of this paper is a real contribution for descriptor LPV systems with significant new results in terms of time-varying fault estimation in spite of disturbances. Both methods presented in [14] or in [3] can only estimate constant fault for descriptor LPV Systems; the real novelty in the paper consists in the ability of the approach to estimate time-varying actuator fault despite disturbances which is a real huge problem and a key problem to solve for some potential extensions to Fault Tolerant Control.

In comparison with the polytopic LPV proportional integral observer studied in [14] which can only estimate constant actuator fault, the main goals of this paper are: to extend existing results about fault estimation of LTI regular systems to polytopic LPV descriptor systems, and also to consider time-varying actuator faults estimation. Sufficient convergence conditions for the APO are given by solving a set of Linear matrix inequalities (LMI).

The outline of this paper is as follows. The structure of the LPV descriptor systems is formulated in Section 2. The Adaptive Polytopic Observer-based actuator fault estimation is presented in Section 3. Finally, in Section 4, a numerical example that considers a LPV descriptor systems is 
used to assess the validity of the proposed approach.

In this paper, the notations are standard. $\mathbf{R}$ denotes the set of real numbers, $\mathcal{C}$ is the complex plane, $A^{+}$denotes the generalized inverse of $A ; Q>0$ or $(Q<0)$ indicates the symmetric matrix $Q$ is positive (or negative) definite; $\|$.$\| denotes the standard norm symbol. Also, \forall$ means "for all". An asterisk $*$ denotes the transposed element in the symmetric position.

\section{PROBLEM STATEMENT}

Let us consider the following LPV descriptor system in fault-free case subject to disturbance:

$$
\left\{\begin{array}{c}
E \dot{x}(t)=\widetilde{A}(\theta(t)) x(t)+\widetilde{B}(\theta(t))(u(t)+f(t))+\widetilde{R}(\theta(t)) d(t) \\
y(t)=C x(t)+H d(t)
\end{array}\right.
$$

where $x(t) \in \mathbf{R}^{n}$ is the state vector, $u(t) \in \mathbf{R}^{p}$ is the inputs vector, $d(t) \in \mathbf{R}^{q}$ is a bounded unknown input vector i.e., $\|d(t)\| \leq \beta$ which represents disturbances and $y(t) \in \mathbf{R}^{m}$ represents the measured outputs vector. $f(t) \in \mathbf{R}^{p}$ represents the actuator fault vector by an additive external signal as in [6], [14]. Sometimes in FDI, these malfunctions can also be represented by a multiplicative external signal as in [33], [24] by the following faulty control input $u_{f}(t)=\left(I_{p}-\gamma\right) u(t)$ which is equivalent to an additive signal such that: $u(t)+f(t)$ where $f(t)=-\gamma u(t)$ with

$$
\begin{aligned}
& \gamma \triangleq \operatorname{diag}\left[\gamma^{1}, \gamma^{2}, \ldots, \gamma^{p}\right], 0 \leq \gamma^{k} \leq 1 \text { such that } \\
& \left\{\begin{array}{l}
\gamma^{k}=1 \rightarrow \text { a total failure of the } k^{t h} \text { actuator } k \in[1, \ldots, p] \\
\gamma^{k}=0 \rightarrow \text { the } k^{t h} \text { healthy actuator }
\end{array}\right.
\end{aligned}
$$

where the term $\gamma^{k}$ represents the loss of effectiveness of $k^{\text {th }}$ actuator. Disturbances and faults are supposed to be unknown. Matrix $E \in \mathbf{R}^{n \times n}$ may be singular and $\operatorname{rank}(E)=r<n$. $\widetilde{A}(\cdot), \widetilde{B}(\cdot)$ and $\widetilde{R}(\cdot)$ are continuous functions which depend affinely on the time-varying parameter vector $\theta(t) \in \mathbf{R}^{l}$. Matrices $C$ and $H$ are fixed. It is also assumed that each component $\theta_{i}(t), i \in[1, \ldots, l]$ of this time-varying parameter vector $\theta(t)$ is bounded and lies into a hypercube such that [30]:

$$
\theta(t) \in \Upsilon=\left\{\theta_{i} \mid \underline{\theta}_{i}(t) \leq \theta_{i}(t) \leq \bar{\theta}_{i}(t)\right\}, \quad \forall i \in[1, \ldots, l], \forall t \geq 0
$$

The matrices $\widetilde{A}(\theta(t)), \widetilde{B}(\theta(t)), \widetilde{R}(\theta(t))$ of the LPV descriptor system (1) with the affine parameter dependence (3) are represented such that $\forall \theta(t) \in \Upsilon$ :

$$
\widetilde{M}(\theta(t))=\widetilde{M}_{0}+\sum_{i=1}^{l} \theta_{i}(t) \widetilde{M}_{i}
$$

where $\widetilde{M}$ stands for matrices $\widetilde{A}, \widetilde{B}$ and $\widetilde{R}$. The system (1) can be transformed into a convex interpolation of the vertices of $\Upsilon$ where the vertices $S_{i}$ of the polytope are defined such that [23]: $S_{i}=\left[\begin{array}{lllll}A_{i}, & B_{i}, & R_{i}, & C & H\end{array}\right], \forall i \in[1, \ldots, h]$ where $h=2^{l}$. The polytopic coordinates are denoted $\rho(\theta(t))$ and vary into the convex set $\Omega$ :

$$
\Omega=\left\{\rho(\theta(t)) \in \mathbf{R}^{h}, \rho(\theta(t))=\left[\rho_{1}(\theta(t)), \ldots, \rho_{h}(\theta(t))\right]^{T}, \rho_{i}(\theta(t)) \geq 0, \forall i, \sum_{i=1}^{h} \rho_{i}(\theta(t))=1\right\}
$$

Then, the usual assumptions related to LPV systems are: $\rho(\theta(t))$ is bounded, it is assumed to be fault-free and it is available. To ease the presentation, it is assumed that the matrices $\widetilde{A}(\cdot), \widetilde{B}(\cdot)$ and $\widetilde{R}(\cdot)$ are given by convex combinations $\forall t \geq 0$ and the polytopic LPV descriptor system with the time-varying parameter vector $\rho(\theta(t)) \in \Omega$ given by:

$$
\left\{\begin{array}{l}
E \dot{x}(t)=\sum_{i=1}^{h} \rho_{i}(\theta(t))\left(A_{i} x(t)+B_{i} u(t)+F_{i} f(t)+R_{i} d(t)\right) \\
y(t)=C x(t)+H d(t)
\end{array}\right.
$$


where $A_{i} \in \mathbf{R}^{n \times n}, B_{i}=F_{i} \in \mathbf{R}^{n \times p}, R_{i} \in \mathbf{R}^{n \times q}, C \in \mathbf{R}^{m \times n}$ and $H \in \mathbf{R}^{m \times q}$ are time invariant matrices defined for the $i^{\text {th }}$ model.

Before starting the design of the APO, we assume that:

A1.

$$
\operatorname{rank}\left(C F_{i}\right)=\operatorname{rank}\left(F_{i}\right)=p \quad \forall i=[1, \ldots, h]
$$

A2. The triple matrix $\left(E, A_{i}, C\right)$ is observable, i.e. [8], [9]

$$
\operatorname{rank}\left[\begin{array}{c}
s E-A_{i} \\
C
\end{array}\right]=n, \forall s \in \mathcal{C}, \quad \forall i=[1, \ldots, h]
$$

and

$$
\operatorname{rank}\left[\begin{array}{c}
E \\
C
\end{array}\right]=n
$$

A3. The fault $f(t)$ satisfies $\|f(t)\| \leq \alpha_{1}$ and the derivative of $f(t)$ with respect to time is norm bounded i.e. $\|\dot{f}(t)\| \leq \alpha_{2}$ and $0 \leq \alpha_{1}, \alpha_{2}<\infty$.

A4.

$$
p+q \leq m
$$

A5.

$$
\operatorname{rank}\left[\begin{array}{cc}
E & 0 \\
C & H
\end{array}\right]=n+\operatorname{rank}(H)
$$

Note that $A 3$ is a quite general assumption in the literature [6], [18]. Assumption $A 4$ has to be verified to be able to provide actuator fault estimation.

The main goal of the paper is to estimate time-varying actuator fault for polytopic LPV descriptor systems. In [32], the authors have performed an adaptive observer so as to estimate time-varying faults but only for regular LTI systems. The authors in [14] have developed a proportional integral observer for actuator fault estimation but with the following constraint $\dot{f}(t)=0$. Moreover, the same constraint has been considered in [3] for discrete-time descriptor systems where the main goal was also to estimate faults. So, in this paper, the main contribution consists in designing an Adaptive Polytopic Observer for LPV descriptor systems which is able to deal with time-varying actuator faults and by the way to tackle the previous restrictive constraints of the above mentioned papers. The following section is dedicated to the design of the Adaptive Polytopic Observer.

\section{ACTUATOR FAULT ESTIMATION FOR POLYTOPIC LPV DESCRIPTOR SYSTEM}

\subsection{Adaptive Polytopic Observer Design}

The proposed Adaptive Polytopic Observer (APO) has the following structure:

$$
\left\{\begin{aligned}
\dot{z}(t) & =\sum_{i=1}^{h} \rho_{i}(\theta(t))\left(N_{i} z(t)+G_{i} u(t)+L_{i} y(t)+F_{i} \hat{f}(t)\right) \\
\hat{x}(t) & =z(t)+T_{2} y(t) \\
\hat{y}(t) & =C \hat{x}(t) \\
r(t) & =S(y(t)-\hat{y}(t)) \\
\dot{\hat{f}}(t) & =\Gamma \sum_{i=1}^{h} \rho_{i}(\theta(t)) U_{i}(\dot{r}(t)+\sigma r(t))
\end{aligned}\right.
$$

where $z(t) \in \mathbf{R}^{n}$ is the observer state vector, $\hat{x}(t) \in \mathbf{R}^{n}$ is the estimated state vector, $r(t) \in \mathbf{R}^{m}$ is the residual vector, $\hat{y}(t) \in \mathbf{R}^{n}$ is the estimated output vector and $\hat{f}(t) \in \mathbf{R}^{p}$ is the estimated actuator fault of $f(t) . N_{i}, G_{i}, L_{i}, T_{2}, U_{i}, S$ are unknown matrices of appropriate dimensions to be determined, $\Gamma \in \mathbf{R}^{p \times p}$ is a learning rate symmetric positive definite matrix and $\sigma \in \mathbf{R}$ is a positive 
scalar. The state estimation error $e(t)$ is defined as:

$$
e(t)=x(t)-\hat{x}(t)
$$

Then, it follows from (6) and (9) that:

$$
e(t)=\left(I_{n}-T_{2} C\right) x(t)-z(t)-T_{2} H d(t)
$$

Under assumption that $\operatorname{rank}\left[\begin{array}{l}E \\ C\end{array}\right]=n$, there exists nonsingular matrices $T_{1} \in \mathbf{R}^{n \times n}$ and $T_{2} \in$ $\mathbf{R}^{n \times m}$ such that [10]:

$$
T_{1} E+T_{2} C=I_{n}
$$

Furthermore, the fault estimation error $e_{f}(t)$ can be expressed as:

$$
e_{f}(t)=f(t)-\hat{f}(t)
$$

From (10), the state estimation error dynamic with the relation (11) is given by:

$$
\dot{e}(t)=T_{1} E \dot{x}(t)-\dot{z}(t)-T_{2} H \dot{d}(t)
$$

Using (6) and (9), the residual $r(t)$ can be expressed as:

$$
r(t)=S C e(t)+S H d(t)
$$

Then, if the following conditions hold true $\forall i=1, \ldots, h$ :

$$
\begin{aligned}
& T_{1} A_{i}-L_{i} C-N_{i} T_{1} E=0 \\
& T_{1} B_{i}-G_{i}=0 \\
& T_{1} E=I_{n}-T_{2} C \\
& T_{1} R_{i}+N_{i} T_{2} H-L_{i} H=0 \\
& T_{2} H=0 \\
& S H=0
\end{aligned}
$$

By taking into account (6), (9) and (13), the estimation error dynamic $\dot{e}(t)$ and the residual $r(t)$ can be written such that:

$$
\begin{aligned}
& \begin{array}{l}
\dot{e}(t)=\sum_{i=1}^{h} \rho_{i}(\theta(t))\left[N_{i} e(t)+M_{i} f(t)+F_{i} e_{f}(t)\right] \\
r(t)=S C e(t) \\
\text { with } \quad M_{i}=\left(T_{1}-I_{n}\right) F_{i}
\end{array}
\end{aligned}
$$

The substitution of (17) into (15) yields to:

$$
\begin{aligned}
& \qquad \begin{array}{l}
N_{i}=T_{1} A_{i}+\left(N_{i} T_{2}-L_{i}\right) C \\
N_{i}=T_{1} A_{i}+K_{i} C
\end{array} \\
& \text { where } \quad K_{i}=N_{i} T_{2}-L_{i}
\end{aligned}
$$

To find simultaneously matrices $T_{1}$ and $T_{2}$ from equations (17) and (19), one can define the following augmented matrix:

$$
\left[\begin{array}{cc}
T_{1} & T_{2}
\end{array}\right]\left[\begin{array}{cc}
E & 0 \\
C & H
\end{array}\right]=\left[\begin{array}{ll}
I_{n} & 0
\end{array}\right]
$$


Under assumption $A 5$, [19], the solution of (27) can be expressed such that:

$$
\left[\begin{array}{ll}
T_{1} & T_{2}
\end{array}\right]=\left[\begin{array}{ll}
I_{n} & 0
\end{array}\right]\left[\begin{array}{cc}
E & 0 \\
C & H
\end{array}\right]^{+}
$$

Matrix $S$ can be determined by solving equation (20) by considering assumption $A 4$ i.e. the number of disturbances and faults can not be larger than the number of measurements, a general solution for (20) is given by (See Chapter 4 of [6]):

$$
S=\Pi_{1}\left[I_{m}-H\left(H^{T} H\right)^{-1} H^{T}\right]
$$

where $\Pi_{1} \in \mathbb{R}^{m \times m}$ is an arbitrary design matrix.

\subsection{Convergence analysis}

Let us consider the following Lemma:

\section{Lemma 1}

Given a scalar $\mu>0$ and a symmetric positive definite matrix $P_{1}$, the following inequality holds [32]:

$$
2 x^{T} y \leq \frac{1}{\mu} x^{T} P_{1} x+\mu y^{T} P_{1}^{-1} y \quad x, y \in \mathbf{R}^{n}
$$

In contrast to the proportional integral observer [14], here time-varying faults are considered. Then, it follows that $\dot{f}(t) \neq 0$ and by consequence, the dynamic of fault estimation error is expressed as follows:

$$
\dot{e}_{f}(t)=\dot{f}(t)-\dot{\hat{f}}(t)
$$

By using (25), the dynamic of the state estimation error (21) becomes:

$$
\dot{e}(t)=\sum_{i=1}^{h} \rho_{i}(\theta(t))[\underbrace{\left(T_{1} A_{i}+K_{i} C\right)}_{N_{i}} e(t)+M_{i} f(t)+F_{i} e_{f}(t)]
$$

The convergence of the state estimation error (32) can be verified by the following Theorem.

\section{Theorem 1}

Under Assumptions $A_{1}$ to $A_{5}$, given scalars $\sigma, \mu>0$, if there exists symmetric positive definite matrices $Q, P_{1}, P_{2}, P_{3}$ and matrices $W_{i}=Q K_{i}$ and $U_{i}$ such that, $\forall i \in[1, \ldots, h]$ :

$$
\left[\begin{array}{cc}
\Psi_{i} & * \\
-\frac{1}{\sigma} F_{i}^{T}\left(Q T_{1} A_{i}+W_{i} C\right) & -\frac{2}{\sigma} F_{i}^{T} Q F_{i}+\frac{1}{\sigma \mu} P_{2}+\frac{1}{\sigma \mu} P_{3}
\end{array}\right]<0
$$

s.t.

$$
\begin{aligned}
W_{i} H+Q T_{1} R_{i} & =0 \\
F_{i}^{T} Q-U_{i} S C & =0
\end{aligned}
$$

where

$$
\Psi_{i}=\left(T_{1} A_{i}\right)^{T} Q+Q\left(T_{1} A_{i}\right)+\frac{1}{\mu} P_{1}+C^{T} W_{i}^{T}+W_{i} C
$$

Then, the following adaptive fault estimation algorithm

$$
\dot{\hat{f}}(t)=\Gamma \sum_{i=1}^{h} \rho_{i}(\theta(t)) U_{i}(\dot{r}(t)+\sigma r(t))
$$

can realize $e(t)$ and $e_{f}(t)$ uniformly bounded, where $\Gamma \in \mathbf{R}^{p \times p}$ is a symmetric positive definite learning rate matrix. 


\section{Proof 1}

Consider the Lyapunov function with the following quadratic form:

$$
V\left(e(t), e_{f}(t)\right)=e^{T}(t) Q e(t)+\frac{1}{\sigma} e_{f}^{T}(t) \Gamma^{-1} e_{f}(t)
$$

Convergence condition for the estimation error yields that the time derivative of the Lyapunov function (37) should be negative definite. The derivative of $V\left(e(t), e_{f}(t)\right)$ with respect to time evaluated on trajectories of the estimation error equation (21) is:

$$
\begin{gathered}
\dot{V}\left(e(t), e_{f}(t)\right)=\sum_{i=1}^{h} \rho_{i}(\theta(t))\left\{e^{T}(t)\left[N_{i}^{T} Q+Q N_{i}\right] e(t)+2 e^{T}(t) Q M_{i} f(t)\right. \\
\left.+2 e^{T}(t) Q F_{i} e_{f}(t)+\frac{1}{\sigma} \dot{e}_{f}^{T}(t) \Gamma^{-1} e_{f}(t)+\frac{1}{\sigma} e_{f}^{T}(t) \Gamma^{-1} \dot{e}_{f}(t)\right\}
\end{gathered}
$$

From (22) and (9), the equation (36) can be expressed as:

$$
\dot{\hat{f}}(t)=\Gamma \sum_{i=1}^{h} \rho_{i}(\theta(t)) U_{i} S(\dot{y}(t)-\dot{\hat{y}}(t)+\sigma(y(t)-\hat{y}(t)))
$$

and under $e(t)=x(t)-\hat{x}(t),(39)$ is equivalent to:

$$
\dot{\hat{f}}(t)=\Gamma \sum_{i=1}^{h} \rho_{i}(\theta(t)) U_{i} S C(\dot{e}(t)+\sigma e(t))
$$

By using (31) and substituting (40) into equation (38), one can obtain:

$$
\begin{gathered}
\dot{V}\left(e(t), e_{f}(t)\right)=\sum_{i=1}^{h} \rho_{i}(\theta(t))\left\{e^{T}(t)\left[N_{i}^{T} Q+Q N_{i}\right] e(t)+2 e^{T}(t) Q M_{i} f(t)+2 e^{T}(t) Q F_{i} e_{f}(t)\right. \\
\left.+\frac{1}{\sigma} \dot{e}_{f}^{T}(t) \Gamma^{-1} e_{f}(t)+\frac{1}{\sigma} e_{f}^{T}(t) \Gamma^{-1}\left[\dot{f}(t)-\Gamma U_{i} S C(\dot{e}(t)+\sigma e(t))\right]\right\}
\end{gathered}
$$

Substituting (21) into (41) and using (35), it follows that:

$$
\begin{gathered}
\dot{V}\left(e(t), e_{f}(t)\right)=\sum_{i=1}^{h} \rho_{i}(\theta(t))\left\{e^{T}(t)\left[N_{i}^{T} Q+Q N_{i}\right] e(t)-\frac{2}{\sigma} e_{f}^{T}(t) F_{i}^{T} Q N_{i} e(t)-\frac{2}{\sigma} e_{f}^{T}(t) F_{i}^{T} Q F_{i} e_{f}(t)\right. \\
\left.-\frac{2}{\sigma} e_{f}^{T}(t) F_{i}^{T} Q M_{i} f(t)+2 e^{T}(t) Q M_{i} f(t)+\frac{2}{\sigma} e_{f}^{T} \Gamma^{-1} \dot{f}(t)\right\}
\end{gathered}
$$

By taking into account assumption $A 3$, for a positive scalar $\mu$ and symmetric matrices $P_{1}>0$, $P_{2}>0$ and $P_{3}>0$ and by using Lemma 1, we can obtain:

$$
\begin{aligned}
2 e^{T}(t) Q M_{i} f(t) & \leq \frac{1}{\mu} e^{T}(t) P_{1} e(t)+\mu f^{T}(t) M_{i}^{T} Q P_{1}^{-1} Q M_{i} f(t) \\
& \leq \frac{1}{\mu} e^{T}(t) P_{1} e(t)+\mu \alpha_{1}^{2} \lambda_{\max }\left(M_{i}^{T} Q^{T} P_{1}^{-1} Q M_{i}\right) \\
\frac{2}{\sigma} e_{f}^{T}(t) \Gamma^{-1} \dot{f}(t) & \leq \frac{1}{\mu \sigma} e_{f}^{T}(t) P_{2} e_{f}(t)+\frac{\mu}{\sigma} \dot{f}^{T}(t) \Gamma^{-T} P_{2}^{-1} \Gamma^{-1} \dot{f}(t) \\
& \leq \frac{1}{\mu \sigma} e_{f}^{T}(t) P_{2} e_{f}(t)+\frac{\mu}{\sigma} \alpha_{2}^{2} \lambda_{\max }\left(\Gamma^{-1} P_{2}^{-1} \Gamma^{-1}\right) \\
\frac{-2}{\sigma} e_{f}^{T}(t) F_{i}^{T} Q M_{i} f(t) & \leq \frac{1}{\mu \sigma} e_{f}^{T}(t) P_{3} e_{f}(t)+\frac{\mu}{\sigma} f^{T}(t) M_{i}^{T} Q F_{i} P_{3}^{-1} F_{i}^{T} Q M_{i} f(t) \\
& \leq \frac{1}{\mu \sigma} e_{f}^{T}(t) P_{3} e_{f}(t)++\frac{\mu}{\sigma} \alpha_{1}^{2} \lambda_{\max }\left(M_{i}^{T} Q^{T} F_{i} P_{3}^{-1} F_{i}^{T} Q M_{i}\right)
\end{aligned}
$$

Using (43), the time derivative of (42) can be bounded as follows:

$$
\begin{gathered}
\dot{V}\left(e(t), e_{f}(t)\right) \leq \sum_{i=1}^{h} \rho_{i}(\theta(t))\left\{e^{T}(t)\left[N_{i}^{T} Q+Q N_{i}\right] e(t)+\frac{1}{\mu} e^{T}(t) P_{1} e(t)-\frac{2}{\sigma} e_{f}^{T}(t) F_{i}^{T} Q N_{i} e(t)\right. \\
\left.-\frac{2}{\sigma} e_{f}^{T}(t) F_{i}^{T} Q F_{i} e_{f}(t)+\frac{1}{\sigma \mu} e_{f}^{T}(t) P_{2} e_{f}(t)+\frac{1}{\sigma \mu} e_{f}^{T}(t) P_{3} e_{f}(t)+\delta\right\}
\end{gathered}
$$


where

$\delta=\max _{i}\left[\mu \alpha_{1}^{2} \lambda_{\max }\left(M_{i}^{T} Q^{T} P_{1}^{-1} Q M_{i}\right)+\frac{\mu}{\sigma} \alpha_{2}^{2} \lambda_{\max }\left(\Gamma^{-1} P_{2}^{-1} \Gamma^{-1}\right)+\frac{\mu}{\sigma} \alpha_{1}^{2} \lambda_{\max }\left(M_{i}^{T} Q^{T} F_{i} P_{3}^{-1} F_{i}^{T} Q M_{i}\right)\right]$

Using (25) and for $W_{i}=Q K_{i}$, the inequality (44) can be written as:

$$
\dot{V}\left(e(t), e_{f}(t)\right) \leq \sum_{i=1}^{h} \rho_{i}(\theta(t))\left\{\left[\begin{array}{ll}
e^{T}(t) & e_{f}^{T}(t)
\end{array}\right] \Xi_{i}\left[\begin{array}{c}
e(t) \\
e_{f}(t)
\end{array}\right]+\delta\right\}
$$

with

$$
\Xi_{i}=\left[\begin{array}{cc}
\Psi_{i} & * \\
-\frac{1}{\sigma} F_{i}^{T}\left(Q T_{1} A_{i}+W_{i} C\right) & -\frac{2}{\sigma} F_{i}^{T} Q F_{i}+\frac{1}{\sigma \mu} P_{2}+\frac{1}{\sigma \mu} P_{3}
\end{array}\right]
$$

and

$$
\Psi_{i}=\left(T_{1} A_{i}\right)^{T} Q+Q\left(T_{1} A_{i}\right)+\frac{1}{\mu} P_{1}+C^{T} W_{i}^{T}+W_{i} C
$$

Considering that $\sum_{i=1}^{h} \rho_{i}(\theta(t))=1$ and $\rho_{i}(\theta(t)) \geq 0$ and because $F_{i}$ is of full column rank $\forall i \in$ $[1, \ldots, h]$ (see assumption $A 1$ ), when $\Xi_{i}<0$ one can obtain that:

$$
\dot{V}\left(e(t), e_{f}(t)\right) \leq-\varepsilon\left\|\left[\begin{array}{c}
e(t) \\
e_{f}(t)
\end{array}\right]\right\|^{2}+\delta
$$

where $\varepsilon=\min _{i}\left(\lambda_{\min }\left(-\Xi_{i}\right)\right)$. Then, $\dot{V}\left(e(t), e_{f}(t)\right)<0$ for $\varepsilon\left\|\left[\begin{array}{c}e(t) \\ e_{f}(t)\end{array}\right]\right\|^{2}>\delta, \forall t \geq 0$ which means that $e(t), e_{f}(t)$ converges to a small set according to Lyapunov stability theory. Now, the obtained gains matrices $K_{i}$ must satisfied also the constraint (18) $\forall i \in[1, \ldots, h]$. Then, the equation (18) can be written as follows:

$$
\left(N_{i} T_{2}-L_{i}\right) H+T_{1} R_{i}=0
$$

and under equation (25), it can be expressed as:

$$
K_{i} H+T_{1} R_{i}=0
$$

For $K_{i}=Q^{-1} W_{i} \forall i \in[1, \ldots, h]$, equation (48) becomes:

$$
W_{i} H+Q T_{1} R_{i}=0
$$

Then, gains matrices $K_{i}$ will be obtained by solving LMIs (33) under constraints (34) and (35).

Therefore, Theorem (1) implies that error $\left(e(t), e_{f}(t)\right)$ are uniformly bounded. Now, its easy to show that the estimated fault can be deduced from the expression (36) as follows :

$$
\hat{f}(t)=\Gamma \sum_{i=1}^{h} \rho_{i}(\theta(t)) U_{i}\left(r(t)+\sigma \int_{t_{f}}^{t} r(\tau) d \tau\right)
$$

where $t_{f}$ is the time of fault occurring. The estimated fault expression (50) combines a proportional term with an integral one. The proportional term have an influence on the rapidity of fault estimation [32]. The proposed APO makes possible to reconstruct the state of the system whatever the presence of the unknown inputs and actuator faults and generate a residual signal which can indicates that a fault occurs.

Remark 1: It is easy to solve inequality (33) of Theorem 1, but the solving difficulty is added due to the presence of equality constraints (34) and (35). However, it is possible to transform (34) and (35) in Theorem 1 into the following optimization problem [7]: 


$$
\begin{gathered}
\text { min } \eta \\
\text { subject to (33) } \\
{\left[\begin{array}{cc}
\eta I & W_{i} H+Q T_{1} R_{i} \\
* & \eta I
\end{array}\right]<0} \\
{\left[\begin{array}{cc}
\eta I & F_{i}^{T} Q-U_{i} S C \\
* & \eta I
\end{array}\right]<0}
\end{gathered}
$$

Remark 2:Note that, after solving the LMI problem under equalities constraints given in Theorem 1 , the input-to-state convergence condition given in (46) is satisfied. Thus, in the case of timevarying faults with a bounded first time derivative, the state estimation error $e(t)$ and the fault estimation error $e_{f}(t)$ converge to a ball, centered at the origin, defined by the terms $\delta$ and $\varepsilon$. The radius of the ball in which $e(t)$ converges can be minimized by a choice of the parameter $\Gamma$ that minimizes $\delta$ without changing $\varepsilon$ (that does not depend on $\Gamma$ ). It thus improves the accuracy of the estimation.

Moreover, the APO studied in this paper can be considered as an improvement of the classical PI observer, in the sense that the convergence of the state estimation error and fault estimation error is proved (in a ball centered at the origin) even in a nonconstant fault case, whereas the assumption of a constant fault is needed to prove the convergence of the state estimation error when using a PI observer [14], [3].

\section{ILLUSTRATIVE EXAMPLE}

The proposed example, considers an electrical network as shown in Figure (1) where $R_{j}, j=$ $1, \ldots, 8$ and $L_{1}$ and $L_{2}$ stand for the resistors and inductors, respectively. $e_{1}(t)$ and $e_{2}(t)$ are the voltage sources which are taken as the control inputs. We denote by $i_{1}(t), i_{2}(t), i_{3}(t)$ and $i_{4}(t)$ the amperage of the currents.

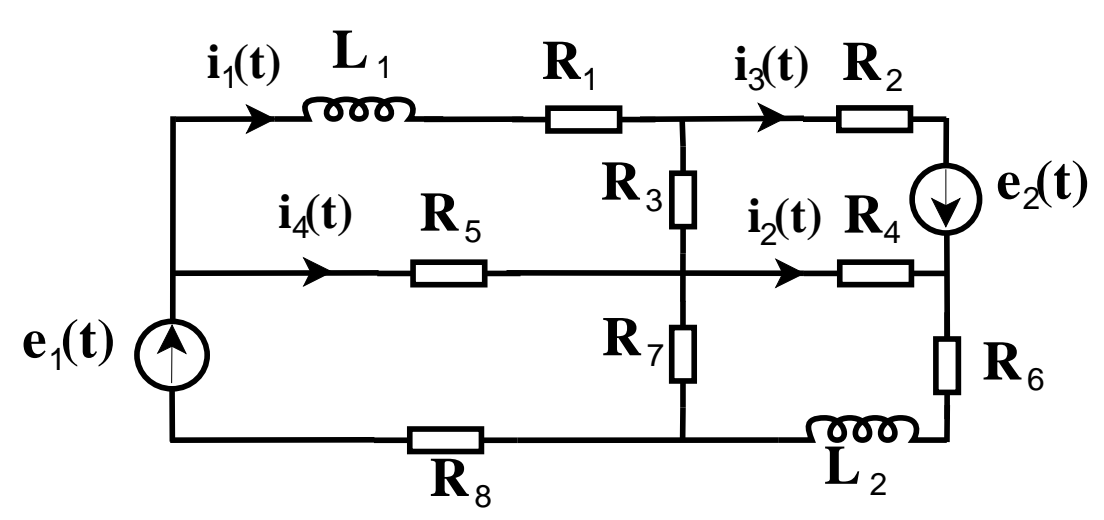

Figure 1. An electrical circuit 
According to the basic circuit theory and the Kirchoff's laws, we get the following DifferentialAlgebraic Equations (DAE) which describe the system:

$$
\begin{aligned}
L_{1} \frac{d i_{1}(t)}{d t} & =-\left(R_{1}+R_{3}+R_{5}\right) i_{1}(t)+R_{3} i_{3}(t)+R_{5} i_{4}(t) \\
L_{2} \frac{d i_{2}(t)}{d t} & =-\left(R_{4}+R_{6}+R_{7}\right) i_{2}(t)+R_{4} i_{3}(t)+R_{7} i_{4}(t) \\
0 & =-\left(R_{2}+R_{3}+R_{4}\right) i_{3}(t)+R_{4} i_{2}(t)+R_{3} i_{1}(t)+e_{1}(t) \\
0 & =-\left(R_{5}+R_{7}+R_{8}\right) i_{4}(t)+R_{7} i_{2}(t)+R_{5} i_{1}(t)+e_{2}(t) \\
y_{1}(t) & =i_{1}(t)+i_{4}(t) \\
y_{2}(t) & =i_{2}(t)+i_{3}(t) \\
y_{3}(t) & =i_{4}(t)
\end{aligned}
$$

Let denote $\left[\begin{array}{llll}x_{1}(t) & x_{2}(t) & x_{3}(t) & x_{4}(t)\end{array}\right]^{T}=\left[\begin{array}{llll}i_{1}(t) & i_{2}(t) & i_{3}(t) & i_{4}(t)\end{array}\right]^{T}$ the state vector, $u(t)=\left[\begin{array}{ll}e 1(t) & e 2(t)\end{array}\right]^{T}$ the control inputs, $y_{1}(t), y_{2}(t)$ and $y_{3}(t)$ the outputs signals, $R_{1}$ and $R_{6}$ two variable resistors. The previous set of DAE can be represented as a LPV descriptor systems as follows:

$$
\left\{\begin{array}{l}
E \dot{x}(t)=\widetilde{A}(\theta(t)) x(t)+\widetilde{B}(\theta(t)) u(t)+\widetilde{R}(\theta(t)) d(t)+\widetilde{F}(\theta(t)) f(t) \\
y(t)=C x(t)+H d(t)
\end{array}\right.
$$

The resulting matrices of the LPV descriptor system are given by:

$$
\begin{gathered}
E=\left[\begin{array}{llll}
1 & 0 & 0 & 0 \\
0 & 1 & 0 & 0 \\
0 & 0 & 0 & 0 \\
0 & 0 & 0 & 0
\end{array}\right], \tilde{B}(\theta(t))=B=\left[\begin{array}{ll}
0 & 0 \\
0 & 0 \\
1 & 0 \\
0 & 1
\end{array}\right], \tilde{F}(\theta(t))=F=B, C=\left[\begin{array}{llll}
1 & 0 & 0 & 1 \\
0 & 1 & 1 & 0 \\
0 & 0 & 0 & 1
\end{array}\right], H=\left[\begin{array}{l}
2 \\
1 \\
1
\end{array}\right] \\
\tilde{A}(\theta(t))=\left[\begin{array}{cccc}
-\frac{R_{11}+\theta_{1}(t)}{L_{1}} & 0 & \frac{R_{13}}{L_{1}} & \frac{R_{14}}{L_{1}} \\
0 & -\frac{R_{22}+\theta_{2}(t)}{L_{2}} & \frac{R_{23}}{L_{2}} & \frac{R_{24}}{L_{2}} \\
R_{31} & R_{32} & -R_{33} & 0 \\
R_{41} & R_{42} & 0 & -R_{44}
\end{array}\right] \text { and } \tilde{R}(\theta(t))=\left[\begin{array}{c}
0 \\
0 \\
0.7+\theta_{1}(t) \\
0.2+\theta_{2}(t)
\end{array}\right]
\end{gathered}
$$

where

$$
\begin{aligned}
& R_{11}=R_{1}+R_{3}+R_{5}=15 \Omega, R_{22}=R_{4}+R_{6}+R_{7}=40 \Omega, \\
& R_{33}=R_{2}+R_{3}+R_{4}=25 \Omega, R_{44}=R_{5}+R_{7}+R_{8}=20 \Omega, \\
& R_{13}=R_{31}=R_{3}=3 \Omega, R_{14}=R_{41}=R_{5}=2 \Omega, \\
& R_{23}=R_{32}=R_{4}=5 \Omega, R_{24}=R_{42}=R_{7}=8 \Omega \\
& \text { and } L_{1}=0.3 \mathrm{H}, L_{2}=0.65 \mathrm{H} .
\end{aligned}
$$

$\tilde{R}$ is a disturbance distribution matrix that affects the system where $d(t)$ is a random vector uniformly distributed in $[-1,1]$ which acts like additive noise. $H$ is also a disturbance distribution matrix that affects the outputs. $\theta_{1}(t)$ and $\theta_{2}(t)$ are two time-varying parameters which vary according to $\theta_{1}(t) \in[-0.5,0.5]$ and $\theta_{2}(t) \in[-1,1]$. The inputs control are described by

$$
\left\{\begin{array}{l}
u_{1}(t)=12 \sin (2.5 t) \\
u_{2}(t)=5
\end{array}\right.
$$

It is assumed that the LPV descriptor system (54) is affected by two actuator faults which followed the assumption $A_{3}$ and that are defined by:

$$
\left\{\begin{array}{l}
\mathrm{f}_{1}(\mathrm{t})=0.35 \mathrm{u}_{1}(\mathrm{t}) \text { occurs in } \mathrm{u}_{1}(\mathrm{t}) \text { when } 8 \leq \mathrm{t} \leq 15 \mathrm{~s} \\
\mathrm{f}_{2}(\mathrm{t})=0.25 \mathrm{u}_{2}(\mathrm{t}) \text { occurs in } \mathrm{u}_{2}(\mathrm{t}) \text { when } 20 \leq \mathrm{t} \leq 30 \mathrm{~s}
\end{array}\right.
$$

Note that different actuator fault can appear in voltage source: an offset for bias or temporal variation for transient fault. Such fault can appear on real source voltage with various temporal duration and different shapes.

The parameters of the polytopic LPV descriptor system evolve in a hyper-rectangle. Consequently, the matrices $A_{i}$ and $R_{i}$ of this system are given by: 


$$
\begin{aligned}
& A_{1}=\left[\begin{array}{cccc}
-51.66 & 0 & 10 & 6.66 \\
0 & -63.07 & 7.69 & 12.3 \\
3 & 5 & -25 & 0 \\
2 & 8 & 0 & -20
\end{array}\right], A_{2}=\left[\begin{array}{cccc}
-51.66 & 0 & 10 & 6.66 \\
0 & -60 & 7.69 & 12.3 \\
3 & 5 & -25 & 0 \\
2 & 8 & 0 & -20
\end{array}\right] \\
& A_{3}=\left[\begin{array}{cccc}
-48.33 & 0 & 10 & 6.66 \\
0 & -63.07 & 7.69 & 12.3 \\
3 & 5 & -25 & 0 \\
2 & 8 & 0 & -20
\end{array}\right], A_{4}=\left[\begin{array}{cccc}
-48.33 & 0 & 10 & 6.66 \\
0 & -60 & 7.69 & 12.3 \\
3 & 5 & -25 & 0 \\
2 & 8 & 0 & -20
\end{array}\right] \\
& R_{1}=\left[\begin{array}{c}
0 \\
0 \\
0.2 \\
-0.8
\end{array}\right], R_{2}=\left[\begin{array}{c}
0 \\
0 \\
0.2 \\
1.2
\end{array}\right], R_{3}=\left[\begin{array}{c}
0 \\
0 \\
1.2 \\
-0.8
\end{array}\right] \text { and } R_{4}=\left[\begin{array}{c}
0 \\
0 \\
1.2 \\
1.2
\end{array}\right]
\end{aligned}
$$

The weighting functions $\rho_{i}(\theta(t))$ are defined as combinations of $\theta_{j}$ and are given by [14]:

$$
\begin{aligned}
& \rho_{1}(\theta(t))=\frac{\theta_{1}(t)-\underline{\theta}_{1}}{\bar{\theta}_{1}-\underline{\theta}_{1}} \frac{\theta_{2}(t)-\underline{\theta}_{2}}{\bar{\theta}_{2}-\underline{\theta}_{2}}=\frac{\left(\theta_{1}(t)+0.5\right)\left(\theta_{2}(t)+1\right)}{2} \\
& \rho_{2}(\theta(t))=\frac{\theta_{1}(t)-\underline{\theta}_{1}}{\bar{\theta}_{1}-\underline{\theta}_{1}} \frac{\bar{\theta}_{2}-\theta_{2}(t)}{\bar{\theta}_{2}-\underline{\theta}_{2}}=\frac{\left(\theta_{1}(t)+0.5\right)\left(1-\theta_{2}(t)\right)}{2} \\
& \rho_{3}(\theta(t))=\frac{\bar{\theta}_{1}-\theta_{1}(t)}{\bar{\theta}_{1}-\underline{\theta}_{1}} \frac{\theta_{2}(t)-\underline{\theta}_{2}}{\bar{\theta}_{2}-\underline{\theta}_{2}}=\frac{\left(0.5-\theta_{1}(t)\right)\left(\theta_{2}(t)+1\right)}{2} \\
& \rho_{4}(\theta(t))=\frac{\bar{\theta}_{1}-\theta_{1}(t)}{\bar{\theta}_{1}-\underline{\theta}_{1}} \frac{\bar{\theta}_{2}-\theta_{2}(t)}{\bar{\theta}_{2}-\underline{\theta}_{2}}=\frac{\left(0.5-\theta_{1}(t)\right)\left(1-\theta_{2}(t)\right)}{2}
\end{aligned}
$$

\subsection{APO design and residual generation}

To design the presented APO according to the proposed methodology, let's verify a necessary assumption $A_{4}$ to be able to provide actuator fault estimation with equation (50). After that, a matrix $S$ (solution of and $S H=0$ ) can be given by (29). Then, let's check the necessary assumption $A_{5}$. Then, matrices $T_{1}$ and $T_{2}$ can be computed from equation (28). After by checking $A_{1}$ and $A_{2}$, the gains matrices can be obtained by solving the LMIs (33) under constraints (34) and (35) via numerical approach within the LMI Toolbox. By choosing the scalars $\sigma=2, \mu=0.5$, these inequalities are fulfilled with:

$$
\begin{gathered}
K_{1}=\left[\begin{array}{ccc}
21.1564 & -17.1376 & -25.1753 \\
-16.1420 & -0.9267 & 33.2106 \\
38.9566 & -18.4702 & -59.4431 \\
23.4193 & -16.5291 & -30.3096
\end{array}\right], K_{2}=\left[\begin{array}{ccc}
1.7542 & 1.8862 & -5.3945 \\
-3.7885 & 13.0178 & -5.4409 \\
5.4732 & -11.0272 & 0.0808 \\
1.7234 & 1.8499 & -5.2967
\end{array}\right] \\
K_{3}=\left[\begin{array}{ccc}
1.4095 & 2.0748 & -4.8937 \\
-2.9091 & 12.5797 & -6.7615 \\
4.2535 & -10.4436 & 1.9365 \\
1.2742 & 2.0773 & -4.6257
\end{array}\right], K_{4}=\left[\begin{array}{ccc}
1.2491 & 2.5999 & -5.0981 \\
-2.7452 & 13.0423 & -7.5518 \\
3.9211 & -10.3360 & 2.4938 \\
1.1244 & 2.5655 & -4.8143
\end{array}\right]
\end{gathered}
$$

The simulation results for time varying actuator faults detection are shown in Figures (2) and (3). From figures (2) and (3), one can see that the residuals are almost zero throughout the time simulation in fault-free case. The residual signals increase in magnitude considerably when actuators faults occur for $t \in[8,15] \mathrm{s}$ and $t \in[20,30] \mathrm{s}$. These two faults can be estimated by using the adaptive fault estimation algorithm as in (50). 


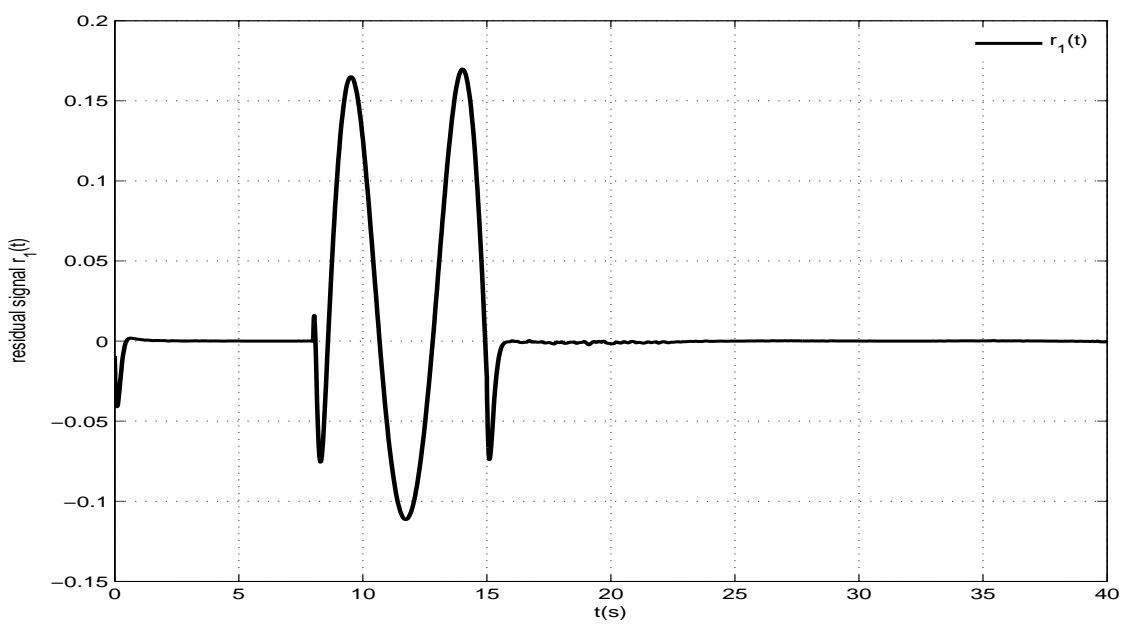

Figure 2. Residual $r_{1}(t)$ signal when a fault occurs in $u_{1}(t)$

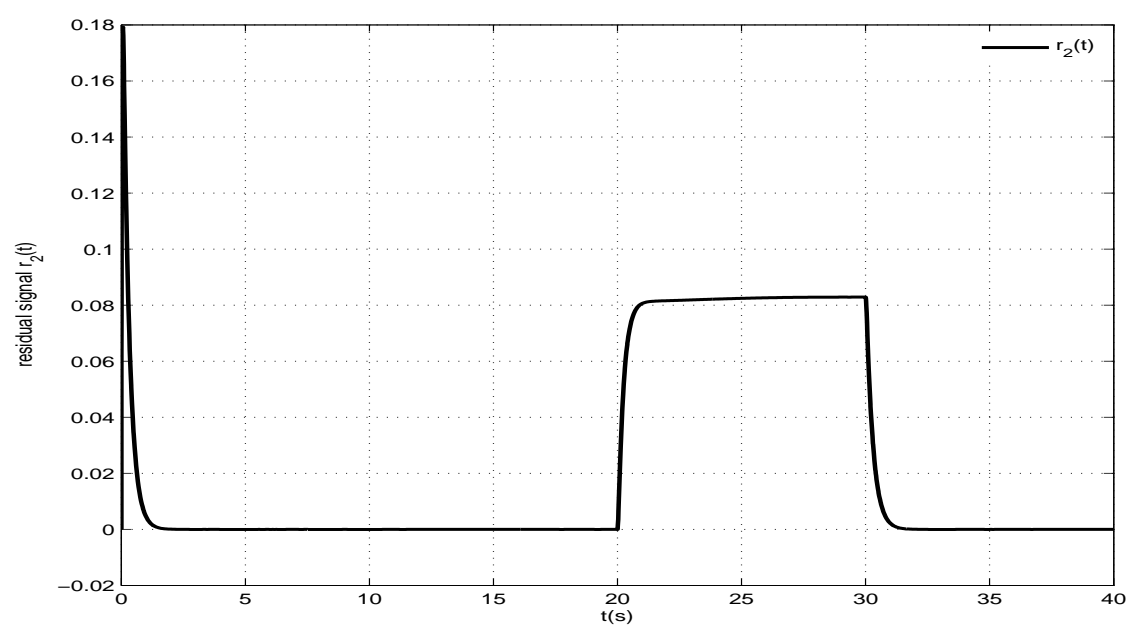

Figure 3. Residual signal $r_{2}(t)$ when a fault occurs in $u_{2}(t)$

\subsection{Actuator fault estimation}

Let recall that $B_{i}=F_{i}=B$. Under assumption $A_{4}$ and by solving the LMIs (33) to (35), getting matrices $U_{i}=U=\left[\begin{array}{ccc}0.8580 & 0.4280 & 0.4263 \\ -8.1460 & -4.0720 & -4.0729\end{array}\right]$ and choosing the learning rate matrix $\Gamma=$ $\operatorname{diag}(1,2)$, the simulation results for time-varying actuator faults estimation using the adaptive algorithm given by (50) are mentioned in the following Figures.

Figures (4) and (5) show that estimated actuator faults by APO can converge to their real values which is more powerful than Proportional Integral Polytopic Observer (PIPO) presented in [14]. Then, it is shown that the PIPO makes possible to decouple the disturbances while it can only detect and estimate constant actuator faults as in Figure (5). Indeed, its actuator fault estimation does not match correctly the real one for the time-varying case in Figure (4). In contrast, the APO shows very good results for both time-varying and constant actuator faults estimations despite the 


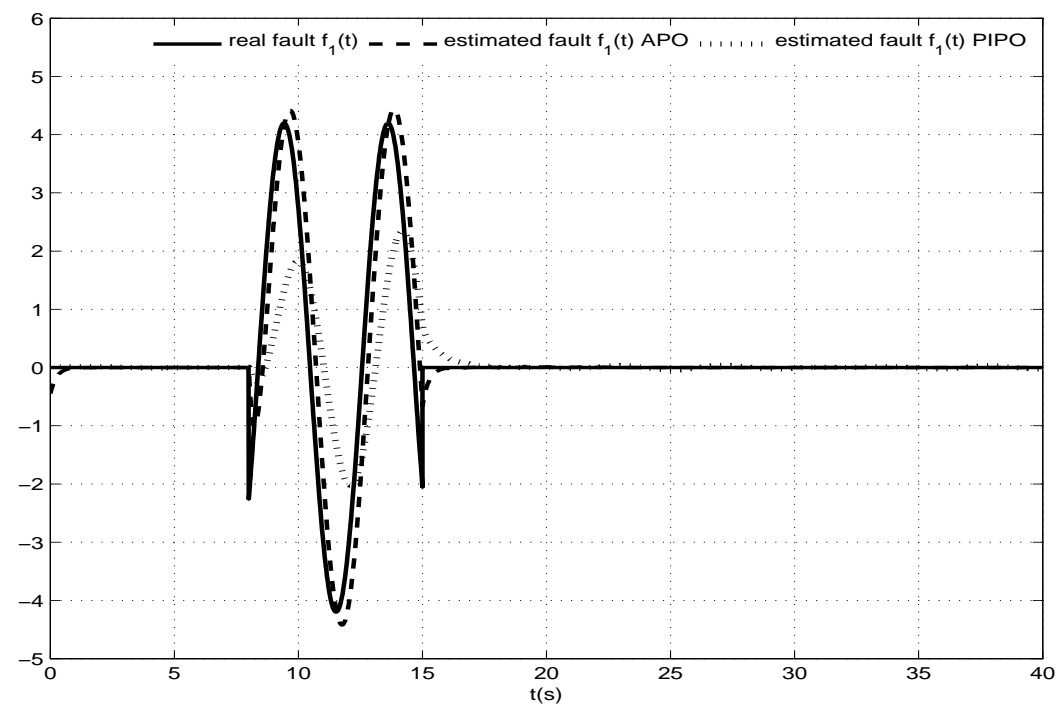

Figure 4. Actuator fault $f_{1}(t)$ and its estimated $\hat{f}_{1}(t)$

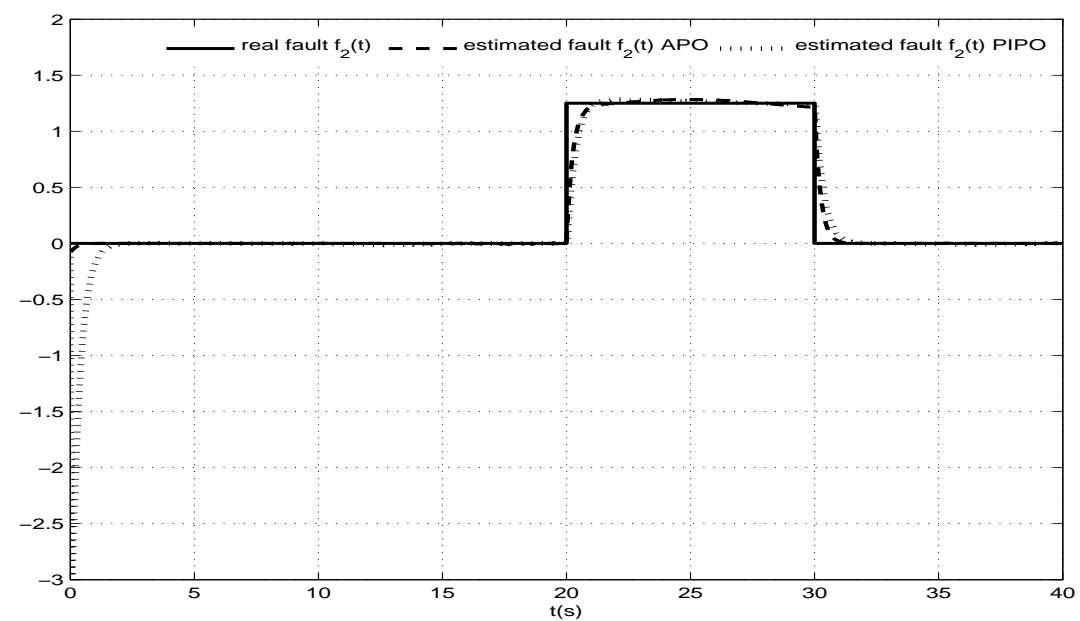

Figure 5. Actuator fault $f_{2}(t)$ and its estimated $\hat{f}_{2}(t)$

presence of disturbance: it is a good improvement for such LPV descriptor systems.

\section{CONCLUSION}

In this paper, an actuator fault estimation scheme based on an Adaptive Polytopic Observer for LPV descriptor systems has been proposed. The developed strategy allows to consider not only constant faults but also time-varying actuator faults. The convergence conditions of this observer has been formulated and solved within a set of LMI under equalities constraints. The developed scheme has been applied in an electrical circuit so as to estimate both time-varying and constant actuator 
faults for polytopic LPV descriptor systems. A comparison with previous works like PI observers, underlines the theoretical and practical improvements for time-varying actuator faut estimation for such LPV descriptor systems.

\section{REFERENCES}

1. Alwi H. and Edwards C. A. Robust Fault Reconstruction for Linear Parameter Varying Systems Using Sliding Mode Observers. International Journal of Robust and Nonlinear Control, DOI: 10.1002/rnc.3009, 2013.

2. Armeni S., Casavola A. and Mosca E. Robust Fault detection and isolation for LPV systems under a sensitivity constraint. International Journal of Adaptive Control and Signal Processing, Vol. 23, no. 1, pp 55-72, 2009.

3. Astorga-Zaragoza C. M., Theilliol D., Ponsart J. C. and Rodrigues M. Fault diagnosis for a class of descriptor Linear Parameter Varying systems. International Journal of Adaptive Control and Signal Processing, Vol. 26, no. 3, pp 208-223, 2012.

4. Bamieh B., Giarre L. Identification of linear parameter varying models International Journal of Robust and Nonlinear Control,Volume 12, Issue 9, pp 841-853, 2002.

5. Bokor J. and G. Balas. Detection filter design for LPV systems-a geometric approach. Automatica, Vol. 40 Issue 3, pp 511-518, 2004.

6. Chen J. and Patton R.J. Robust model-based fault diagnosis for dynamic systems Kluwer Academic Publishers, 1999.

7. Corless M.and Tu J. State and Input Estimation for a Class of Uncertain Systems Automatica, Vol. 34, No. 6, pp. 757-764, 1998

8. Dai L. Singular Control Systems Springer, Germany, 1989.

9. Duan G-R. Analysis and Design of descriptor Linear Systems Advances in Mechanics and Mathematics Springer,2010.

10. Darouach M. and Boutayeb M. Design of Observers for Descriptor Systems IEEE transactions on Automatic Control, vol no. 40, pp 1323-1327, 1995.

11. De Lira S., Puig V., Quevedo J. and Husar A. LPV observer design for PEM fuel cell system: Application to fault detection Journal of Power Sources, 196, pp 4298-4305, 2011.

12. De. Oca S. M., Rotondo D.,Nejjari F.,Puig V. Fault estimation and virtual sensor FTC approach for LPV systems, In proceedings: 50th IEEE Conference on Decision and Control and European Control Conference (CDC-ECC), CD-Rom, Orlando, USA, 2011.

13. Grenaille S., Henry D., Zolghadri A. A method for designing Fault Diagnosis Filters for LPV polytopic systems Journal of Control Science and Engineering , 2008.

14. Hamdi H., Rodrigues M., Mechmeche C., Theilliol D. and BenHadj Braiek N., Fault Detection and Isolation in Linear Parameter Varying Descriptor systems via Proportional Integral Observer. International journal of adaptive control and signal processing, Vol. 26, No. 3, pp 224-240, 2012.

15. Hamdi H., Rodrigues M., Mechmeche C. and BenHadj Braiek N. Robust Fault Detection and Estimation for Descriptor Systems Based on Multi-Models Concept International Journal of Control, Automation and Systems, Vol. 10, no. 6, pp 1260-1266, December 2012.

16. Hamdi H., Rodrigues M., Mechmeche C., Theilliol D. and BenHadj Braiek N. State Estimation for Polytopic LPV Descriptor Systems: Application to Fault Diagnosis. The 7th IFAC Symposium on Fault Detection, Supervision and Safety of Technical Processes, Barcelona, Spain, pp 438-443, 2009.

17. Jiang B., Zhang K., and Peng S. Integrated fault estimation and accommodation design for discrete-time TakagiSugeno fuzzy systems with actuator faults. IEEE Transactions on Fuzzy Systems, vol. 19, no. 2, pp 291-304, 2011.

18. Jiang B., Staroswiecki M. and Cocquempot V. Fault Accommodation for Nonlinear Dynamic Systems. IEEE Transactions on Automatic Control, Vol. 51, No. 9, Sept 2006.

19. Koenig D. Unknown Input Proportional Multiple-Integral Observer Design for Linear Descriptor Systems: Application to State and Fault Estimation. IEEE Transactions on Automatic control, Vol 50, no.2, pp 212-217, February 2005.

20. F. L. Lewis. A Survey of Linear Singular Systems Circuits Systems and Signal Processing,vol. 5, pp 3-36, 1986.

21. Mattson S., Elmqvist H., and Otter M. Physical system modeling with modelica. Control Engineering Practice, vol. 6, pp 501-510, 1998.

22. Puig V., Quevedo J., Escobet T., Nejjari F., De Las Heras S. Passive Robust Fault Detection of Dynamic Processes Using Interval Models IEEE Transactions on Control Systems Technology, Vol. 16, Issue 5, 2008.

23. Rodrigues M., Theilliol D. and Sauter D. Design of an Active Fault Tolerant Control for Nonlinear Systems described by a Multi-Model Representation. Proc. The 20th IEEE International Symposium on Intelligent Control (ISIC'05) and the 13th Mediterranean Conference on Control and Automation (MED'05), Limassol, Cyprus, pp 1579-1584, 2005.

24. Rodrigues M., Theilliol D., Aberkane S. and Sauter D. Fault Tolerant Control Design for Polytopic LPV Systems. International Journal of Applied Mathematics and Computer Sciences, Vol. 17, No.1, pp 27-37, 2007.

25. Rodrigues M., Sahnoun M., Theilliol D., Ponsart J.-C. Sensor Fault Detection and Isolation Filter for Polytopic LPV Systems: A Winding Machine Application Journal of Process Control, Volume 23, Issue 6, pp 805-816, 2013.

26. Rotondo D.,Nejjari F., Puig V. Quasi-LPV modeling, identification and control of a twin rotor MIMO system. Control Engineering Practice, Vol 21(6), pp 829-84, 2013.

27. Wang Z.,Shen Y.,Zhang X. and Wang Q. Observer design for discrete-time descriptor systems: An LMI approach. Systems \& Control Letters Vol. 61,pp 683-687, 2012.

28. Wang H. and Daley S. Actuator fault diagnosis: An adaptive observer-based technique. IEEE Transactions on Automatic Control, Vol. 41, no. 7, pp 1073-1078, 1996.

29. Wei, X. and Verhaegen, M. LMI solutions to the mixed $H_{-} / H_{\infty}$ fault detection observer design for linear parametervarying systems Int. J. Adapt. Control Signal Process, Vol. 36, no. 2, pp 114-136, 2011. 
30. Wu F. Control of Parameter Varying Systems PhD Thesis, University of California at Berkeley, USA, 1995.

31. Zhang K. and Jiang B. Dynamic output feedback fault tolerant controller design for Takagi-Sugeno fuzzy systems with actuator faults. IEEE Transactions on Fuzzy Systems, vol. 18, no. 1, pp 194-201, 2010.

32. Zhang K., Jiang B., and Cocquempot V. Adaptive Observer-based Fast Fault Estimation. International Journal of Control, Automation, and Systems, vol. 6, no. 3, pp 320-326, 2008.

33. Zhang Y.,Jiang J. Design of Integrated Fault Detection, Diagnosis and Reconfigurable Control Systems, Proceedings of the 38th IEEE Conference on Decision and Control (CDC) Phoenix, Arizona, USA 1999.

34. Zhang Y.,Jiang J. Bibliographical review on reconfigurable fault-tolerant control systems, Annual Reviews in Control, 32,pp 229-252, 2008. 